\title{
A Taxonomical Review of Multiplexer Designs for Electronic Circuits \& Devices
}

\author{
Malti Bansal ${ }^{*}, 1$, Harmandeep Singh ${ }^{2}$, Gaurav Sharma ${ }^{2}$ \\ ${ }^{1}$ Department of Electronics \& Communication Engineering, \\ Delhi Technological University (DTU), Delhi-110042, India. \\ ${ }^{2}$ Department of Information Technology, \\ Delhi Technological University (DTU), Delhi-110042, India. \\ *maltibansal@gmail.com
}

\begin{abstract}
This research paper reviews and briefly discusses about the multiplexers and demultiplexers. This research paper aims to explore the history of multiplexers, types of multiplexers, applications and the real-time use cases of multiplexers. Furthermore, it also includes a brief introduction on the different multiplexing techniques employed in analog and digital electronics, ongoing research studies and future research scope for multiplexers.
\end{abstract}

Keywords - Multiplexers, demultiplexers, study, comparison.

\section{INTRODUCTION}

In electronics, often there is a requirement to combine several analog signals or digital data streams, which combine into one or select one from several input data lines in order to forward the intended input to the single output line. The purpose to save expensive resources, as many input data lines or streams can make use of only one shared resource or device, it makes the circuit to work more efficiently. This technique or method is known as "multiplexing" or simply "muxing". Converse to "muxing" is the technique or method called "demultiplexing" or simply "demuxing". The word "demultiplex" means one to many, demuxing is the method used to obtain data from one input line and transmit it to several output lines, where transmission is controlled by select lines. Thus, a multiplexer can be considered as a switch with multiple-inputs and single-output, while demultiplexer is considered as a switch with a single-input and multiple-outputs. Very often on the receiving end, where a multiplexer is combined with a complementary demultiplexer [1-3]. 


\section{HISTORY}

Today, multiplexing is most commonly used in telecommunication industries. Multiplexing originated back in the 1870s in telegraphy, when telecommunication was in its initial stages. In 1910 George Owen Squier developed a telephone carrier multiplexing.[1] During early periods of telegraphy, it was really expensive and difficult to lay out the long telegraphic cables, and utilization of the signal line to their full efficacy was not possible, therefore there is a need to develop a method for full utilization of transmission cables. In order to overcome this problem, one of the first multiplexing systems in telegraphy was developed by Baudot and Delany, which allowed multiple telegraphists to share a single line.[4] Jean-MauriceÉmile Baudot patented his telegraphic multiplexing technique in 1874. This paved way for further improvement and development in multiplexing techniques and multiplexer devices in telegraphy and then in telephony. The first research paper, which reported the term 'multiplexer' was written by anonymous, the paper is called "Anonymous, On the synchronous-multiplex telegraph, Proc. Am. Philos. Soc. 21(114) (1883), pp. 326-328”. Some of the early experimental works in telephony multiplexing were done by Willard Miner in the 1900s. But the landmark work in this field was done by Herbert Raabe (1909-2004) and Claude Shannon (1916-2001) during the 1940s, [4] which proved to be pioneering work for the improvement and development of new multiplexing techniques in the years to come both in analog electronics and digital electronics.

\section{MulTiPLEXER}

\section{A. What is a multiplexer?}

A multiplexer is a device that converts parallel data into serial data by routing data from many input lines/streams to one output line/stream. Multiplexers are used in both analog and digital electronics. In analog circuits, relays and transistors make up a multiplexer, while in digital circuits multiplexers are developed by using logic gates [1]. Digital multiplexers have signal lines that can be used to steer data from any input line to the output line. Commonly used digital multiplexers are 2-to-1, 4-to-1, 8-to-1, 16-to-1 multiplexers etc. 


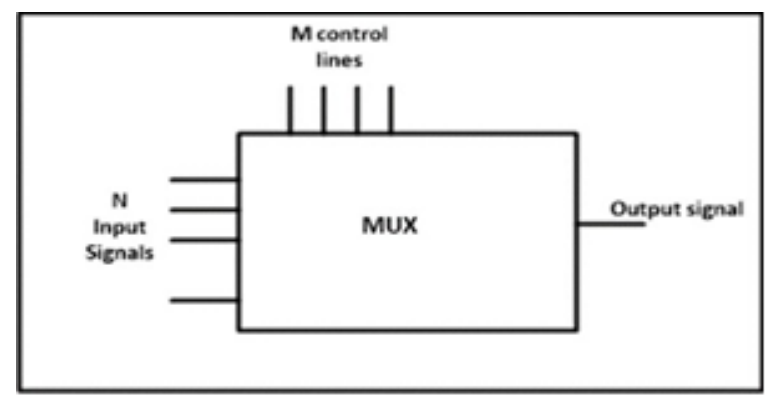

Fig. 1: Multiplexer Pin Diagram

(Reproduced from [1])

A 4-to-1 multiplexer can be used to explain the working of a digital multiplexer. The 4-to-1 multiplexer has four input bits, two control bits, and one output bit. Fig. 1 shows "Multiplexer pin diagram".

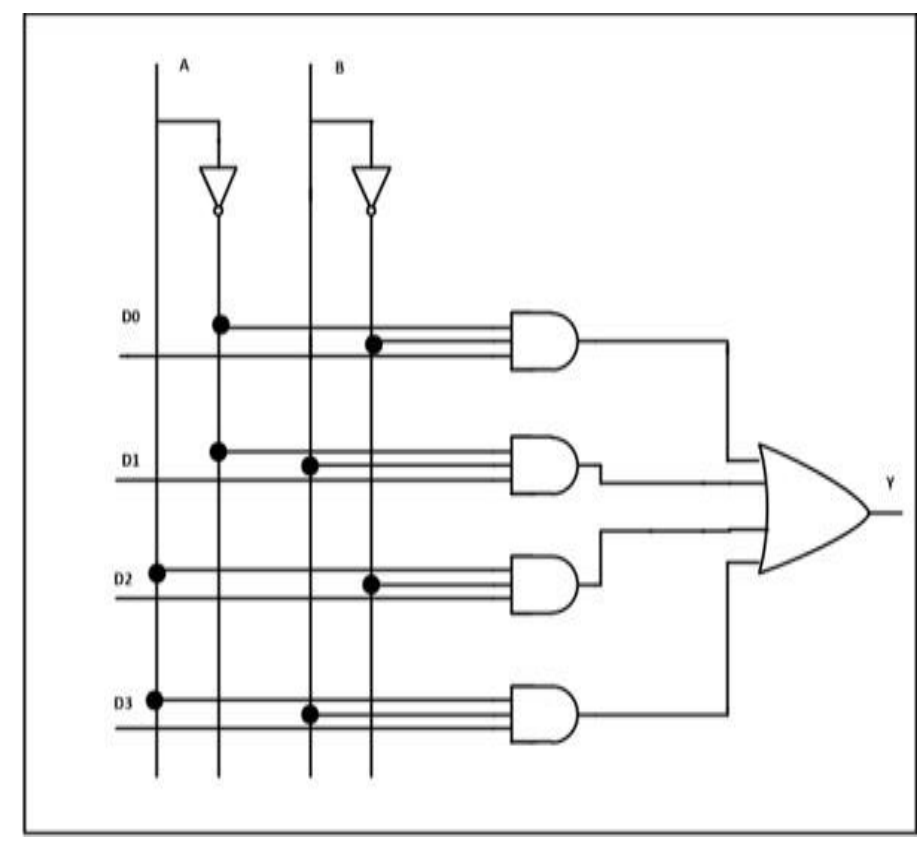

Fig. 2: 4-to-1 Multiplexer circuit diagram

Here the 4 input bits are D00, D01, D02, and D03. Only one of these is forwarded to the output $\mathrm{Y}$. The transmission is constrained by the control lines $\mathrm{A}$ and $\mathrm{B}$, whose values determine which info bit is to be communicated to output Y. For instance, assuming the estimation of $\mathrm{AB}$ is 00 , the first AND gate is activated while the rest are not enabled and the info bit from D00 is sent to output Y. Table 1 shows "Truth Table for 4-to-1 multiplexer". 
Table 1: Truth Table for 4-to-1 multiplexer

\begin{tabular}{|l|l|l|}
$A$ & B & Y \\
\hline 0 & 0 & D00 \\
\hline 0 & 1 & D01 \\
\hline 1 & 0 & D02 \\
\hline 1 & 1 & D03
\end{tabular}

In a combinational circuit, a multiplexer circuit is very similar to an encoder circuit and can be built using AND, OR, NOT gates. Fig. 2 shows a "4-to-1 Multiplexer circuit diagram".

\section{B. Types of multiplexers}

Common types of digital multiplexers are:

- 2 to 1 multiplexer (with 1 select line)

- 4 to 1 multiplexer (with 2 select lines)

- 8 to 1 multiplexer (with 3 select lines)

- 16 to 1 multiplexer (with 4 select lines)

By combining different multiplexers, we can make any desired $\mathrm{m}$ to 1 multiplexer.

\section{Advantages/Disadvantages of a multiplexer}

- Advantages: By using multiplexers, combinational circuits can be made cost-efficient, less complex, less usage of wiring, and sharing of resources.

- Disadvantages: There is an additional delay because of switching ports and additional I/O signals used for controlling the MUX.

\section{Applications of multiplexers [1]}

- In communication systems: Multiplexers help handle multiple transmission channels/lines, it allows the transfer of different formats of data such as audio, video streams simultaneously over a single line. Now the system is more efficient because of the use of multiplexers in building the logic.

- In telephony: Helps in sharing and efficient utilization of transmission medium i.e., telephone cables.

- In computer memory: Diminishes the number of memory paths needed to interface different PC parts to the memory. 
- In GPS (global positioning system) technology for the satellite to ground communication [6].

\section{Demultiplexer}

\section{A. What is a demultiplexer?}

A demultiplexer is a device that converts serial data into parallel data by routing data from one input line/stream to many output lines/streams. Demultiplexers are used both in analog and digital electronics. In analog circuits relays and transistors make up a demultiplexer while in digital circuits demultiplexer is made using logic gates [1]. Digital demultiplexers have signal lines that can be used to steer data from the input line to any output line. Commonly used digital demultiplexers are 1-to-2, 1-to-4, 1-to-8, 1-to-16 demultiplexers etc. Fig. 3 shows "Demultiplexer pin diagram".

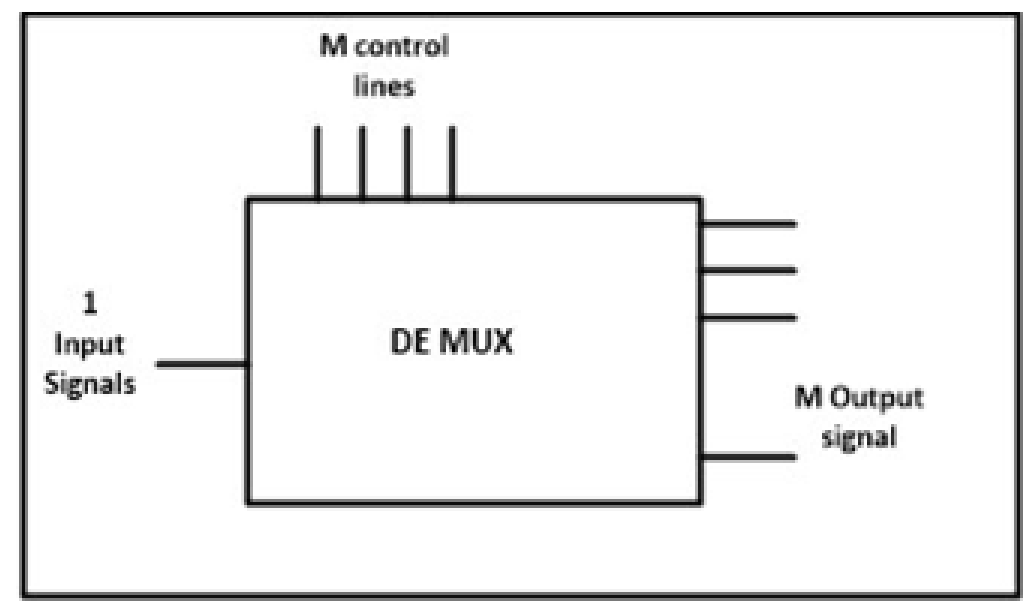

Fig. 3: Demultiplexer pin diagram (Reproduced from [1])

A 1-to-4 demultiplexer can be used to explain the working of a digital demultiplexer. 1-to4 multiplexers have one input bit, two control bits, and four output bits. 


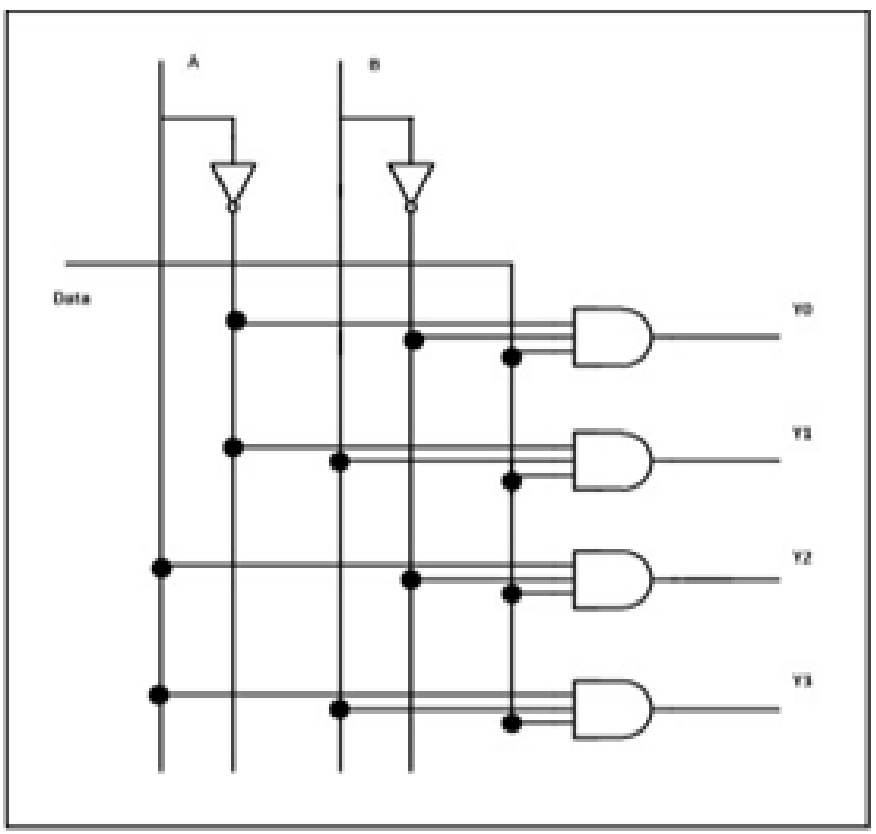

Fig. 4: 1-to-4 Demultiplexer circuit diagram (Reproduced from [1])

Here the data input bit is D. It is forwarded to one of the four output lines Y0, Y1, Y2, and Y3. The transmission is controlled by the control lines $\mathrm{A}$ and $\mathrm{B}$, whose values determine which input bit is to be transmitted to output Y. For example, if the value of $A B$ is 01 , then the second AND gate are activated while the rest are not enabled and the input bit i.e., D is transmitted to output i.e., Y1. Table 2 shows "Truth table for a 1-to-4 demultiplexer".

Table 2: Truth table for a 1-to-4 demultiplexer

\begin{tabular}{|l|l|l|}
\hline$A$ & B & $Y$ \\
\hline 0 & 0 & $Y 0=D$ \\
\hline 0 & 1 & $Y 1=D$ \\
\hline 1 & 0 & $Y 2=D$ \\
\hline 1 & 1 & $Y 3=D$
\end{tabular}

In a combinational circuit, a demultiplexer circuit is very similar to a decoder circuit and can be built using AND, OR, NOT gates. Fig. 4 shows "1-to-4 Demultiplexer circuit diagram". 


\section{B. Types of demultiplexers}

Digital demultiplexers' common types are:

- 1 to 2 demultiplexer (with 1 select line)

- 1 to 4 demultiplex er (with 2 select lines)

- 1 to 8 demultiplex er (with 3 select lines)

- 1 to 16 demultiplexer (with 4 select lines)

By combining different demultiplexers, we can make any desired 1 to m demultiplexer.

\section{Advantages/Disadvantages of a Demultiplexer}

- Advantages: A combination of MUX and DEMUX enhances the efficiency of the communication system. DEMUX divides the mutual signals back into their individual streams. DEMUX also acts as decoders for many security systems.

- Disadvantages: Synchronization of different signals causes the wastage of bandwidth and causes signal delays in the circuit.

\section{Applications of Demultiplexers [1]}

- In communication systems: In combination with multiplexers demultiplexers help in better transmission and reception of simultaneous data streams like audio and video in a communication system.

- In an $A L U$ (arithmetic logic unit): Here a demultiplexer is associated with numerous registers or other stockpiling gadgets while the output of the ALU goes about as input for demultiplexer. In this way, the input of ALU is stored in various areas in memory.

- In serial to parallel convertor: Serial data signals are first fed to a demultiplexer, which stores data at different locations and later on it can be read parallelly [5].

- Helps in connecting single sources to multiple destinations.

\section{V.Multiplexing Technologies/TeChniQues}

The method of communicating different signals which either might be analog or digital over a solitary output medium is known as multiplexing. 
Some examples of different types of multiplexing technologies/techniques are [7]:

- Frequency Division Multiplexing (FDM)

- Orthogonal Frequency Division Multiplexing (OFDM)

- Wavelength Division Multiplexing (WDM)

- Dense Wavelength Division Multiplexing (DWDM)

- Conventional Wavelength Division Multiplexing (CWDM)

- Reconfigurable Optical Add-Drop Multiplexer (ROADM)

- Add/Drop Multiplexing (ADM)

- Inverse Multiplexing (IMUX).

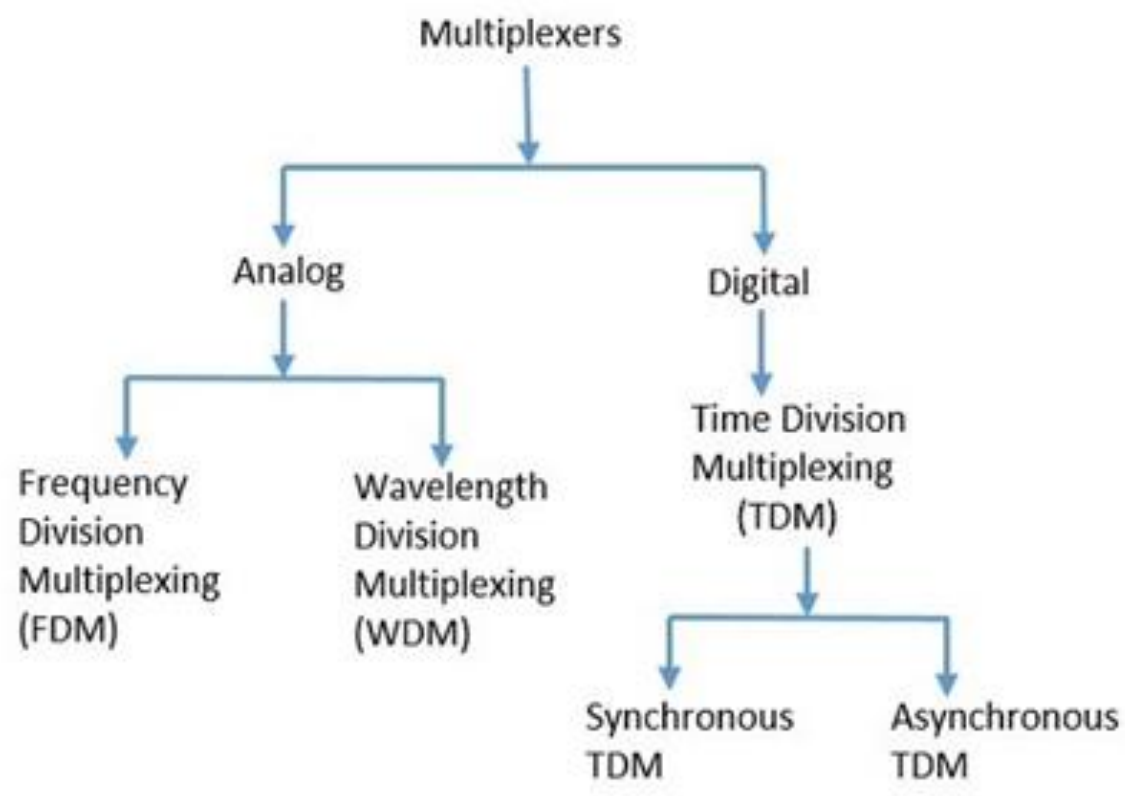

Fig. 5: Multiplexing Techniques

(Reproduced from [7])

Fig. 5 shows various analog and digital electronics based multiplexing techniques. Some multiplexing techniques are briefly explained: 
Journal of Electronics and Informatics (2021)

Vol.03/ No.02

Pages: $77-88$

https://www.irojournals.com/iroei/

DOI: https://doi.org/10.36548/jei.2021.2.001

- Time Division Multiplexing: In this, the signal from various low bit rate channels are communicated over a solitary high bit rate channel by assigning each low bit rate channel a time slot on a high bit rate channel. Data is sent segmentally during particular time intervals[8][9].

- Statistical Multiplexing: This multiplexing technique allows seamless real-time transmission of audio-video data streams by bundling different bit rate data stream signals into a single bandwidth signal. It combines smaller bits with larger bits.[9]

- Inverse Multiplexing: Inverse multiplexing is the opposite of statistical multiplexing, in this larger signal is broken into smaller bits to be transmitted over multiple channels.[9]

- Space Division Multiplexing: In space division multiplexing, with an end goal to restrict the impedance or commotion, information/data from different streams is scattered across the same wire or medium.[9]

- Frequency Division Multiplexing: In this type of multiplexing, optimum utilization of bandwidth is aimed at transmitting each piece of data at a different level of frequency across the same bandwidth. Thus, the entirety of bandwidth is simultaneously occupied by different signals at different positions.[9]

- Wavelength Division Multiplexing: Wavelength division multiplexing is usually employed in fiber optics communication as WDM depends upon the shared medium, which in this case is optic fiber. Here individual wavelengths are used to transmit individual signals [9].

\section{Ongoing Study \& Future ScOPE OF MultiPlexers/MultiPlexing}

Advances in multiplexing technology are a wide area of research at the moment. work on transmission technology via fiber optics especially is an area where future developments are looking good. Commonplace electrical-based multiplexing, for example , electrical timedivision multiplexing and cycle division multiplexing seem to fail to satisfy the prerequisites of current/modern fiber-optic communication [10]. Multi slot amplitude coding is the most recent multiplexing procedures, which is proposed as an option in contrast to regular ETDM and DCDM. In addition to that, improvements in spectral efficiency which lie outside the capability of ETDM have been consistently demonstrated and pier-reviewed in [10]. MSAC signal additionally takes into account extraction of $10 \mathrm{ghz}$ clock signal which is 
Journal of Electronics and Informatics (2021)

Vol.03/ No.02

Pages: $77-88$

https://www.irojournals.com/iroei/

DOI: https://doi.org/10.36548/jei.2021.2.001

significant for the recovery circuit. Simulation arrangement of MSAC in optical communication framework comprises of $\mathrm{n}$ beat design generators, MSAC multiplexer, transmitter segment, transmission segment, a receiver section, external modulator, and a persistent wave laser are segments of the transmitter area. the transmission segment comprises an optical attenuator, optical fiber, the input signal in the form of a synchronized binary data stream is obtained by the clock signal of each pulse generator for the MSAC multiplexer [10]. The receiver segment is comprised of the optical intensifier, PIN photodiode, electrical lowpass filter and clock, and information/data recuperation [10]. MSAC system execution is discovered to be superior to DCDM, in terms of receiver sensitivity, spectral width, efficiency, optical signal to noise ratio, and chromatic dispersion tolerance [10-27].

\section{REFERENCES}

[1] Dalal, Ankit and Ankur Atri. "A General Overview of Multiplexer and Demultiplexer." International Journal of Research 1 (2014): 1293-1298.

[2] Dean, Tamara (2010). Network+ Guide to Networks. Delmar. pp. 82- 85.

[3] Debashis, De (2010). Basic Electronics. Dorling Kindersley. p. 557. ISBN 9788131710685.

[4] Butzer, P. L., Dodson, M. M., Ferreira, P. J. S. G., Higgins, J. R., Lange, O., Seidler, P. and Stens, R. L.(2011) 'Multiplex signal transmission and the development of sampling techniques: the work of Herbert Raabe in contrast to that of Claude Shannon', Applicable Analysis, 90: 3, $643-688$

[5] Bates, Regis J; Bates, Marcus (2007), Voice and Data Communications, ISBN 9780072257328

[6] Lipták, Béla (2002). Instrument engineers' handbook: Process software and digital networks.CRC Press. p. 343.

[7] Admin. (2021, January 5). What are Multiplexer and Types of Multiplexing Techniques? WatElectronics.com. https://www.watelectronics.com/what-is-multiplexer-and-types/

[8] Admin. Types of $\quad$ Multiplexers. https://www.thomasnet.com/articles/automation-electronics/multiplexer-types/

[9] Admin. Multiplexer Design and Performance. ThomasNet. https://www.thomasnet.com/articles/automation-electronics/multiplexer-design/ 
Journal of Electronics and Informatics (2021)

Vol.03/ No.02

Pages: $77-88$

https://www.irojournals.com/iroei/

DOI: https://doi.org/10.36548/jei.2021.2.001

[10] Rahmat Talib and Mohammad Faiz Liew Abdullah. (2017) "The Future Electrical Multiplexing Technique for High-Speed Optical Fibre", DOI: 10.5772/intechopen.68407.

[11] https://www.intechopen.com/books/optical-fiber-and-wireless-communications/thefuture-electrical-multiplexing-technique-for-high-speed-optical-fibre.

[12] M. Bansal and J. Singh, "Qualitative Analysis of CMOS Logic Full Adder and GDI Logic Full Adder using 18 nm FinFET Technology," 2019 3rd International Conference on Recent Developments in Control, Automation \& Power Engineering (RDCAPE), Noida, India, 2019, pp. 404-407, doi: 10.1109/RDCAPE47089.2019.8979087.

[13] N. Raghav and M. Bansal, "Analysis of Power Efficient 6-T SRAM Cell with Performance Measurements," 2017 International Conference on Innovations in Control, Communication and Information Systems (ICICCI), Greater Noida, India, 2017, pp. 1-4, doi: 10.1109/ICICCIS.2017.8660819.

[14] M. Rawat, M. Bansal, “ Voltage Mode Two-Phase and Four-Phase Sinusoidal Oscillator using VDCC", International Journal of Advanced Research in Electronics and Communication Engineering, 2015, Vol. 4, Issue 6, pp. 1687-1691.

[15] D. Ruhela, M. Bansal, "Adiabatic Vedic Multipler Design Using Chinese Abacus Approach", International Journal of Advanced Research in Computer and Communication Engineering, Vol. 4, Issue 4, pp. 81-85, 2015.

[16] M. Bansal, D. Ruhela, "High Speed and Area Efficient Vedic Multiplier using Adiabatic Logic", Journal of Basic and Applied Engineering Research, Vol. 1, Issue 11, pp. 14-17, 2014.

[17] N. Raghav, M. Bansal, "Analytical Study of Full Adder Circuit using Modified Glitch Free Cascadable Adiabatic Logic", International Conference on Advanced Production and Industrial Engineering (ICAPIE-2017), 2017.

[18] M. Bansal and J. Singh, "Qualitative Analysis of 2-bit CMOS Magnitude Comparator and GDI Magnitude Comparator using FinFET Technology (18nm)," 2020 International Conference on Smart Electronics and Communication (ICOSEC), Trichy, India, 2020, pp. 1323-1327, doi: 10.1109/ICOSEC49089.2020.9215251.

[19] M. Bansal and J. Singh, "Comparative Analysis of 4-bit CMOS Vedic Multiplier and GDI Vedic Multiplier using 18nm FinFET Technology," 2020 International Conference on Smart Electronics and Communication (ICOSEC), Trichy, India, 2020, pp. 1328-1332, doi: 10.1109/ICOSEC49089.2020.9215317. 
Journal of Electronics and Informatics (2021)

Vol.03/ No.02

Pages: $77-88$

https://www.irojournals.com/iroei/

DOI: https://doi.org/10.36548/jei.2021.2.001

[20] M. Bansal, H. Saxena, "Power-Delay Behaviour of Digital Circuits Designed using MOSFET, CNTFET and FinFET, with the Scope of Miniaturization in these Transistors", International Journal of Advanced Research in Computer and Communication Engineering (IJARCCE), Vol. 9, Issue 2, pp. 64- 71, 2020.

[21] M. Bansal, H. Saxena, "Comparative Analysis and Behavior of Digital Applications simulated using MOSFET, CNTFET and FinFET Transistors in HSPICE", International Journal of Advanced Research in Computer and Communication Engineering (IJARCCE), Vol. 9, Issue 2, pp. 54-58, 2020.

[22] M. Bansal, H. Saxena, "High Speed and Low Power Consumption Multipliers using FinFET Technology", International Journal of Advanced Research in Computer and Communication Engineering (IJARCCE), Vol. 9, Issue 2, pp. 59-63, 2020.

[23] M. Bansal, J. Singh, "CMOS MOSFET, FinFET and CNTFET: Qualitative Analysis", National Conference on Emerging Trends in Electronics \& Communication-2019, pp. 79-84, 2019.

[24] N. Raghav and M. Bansal, "Analytical study of high performance flip-flop circuits based on performance measurements," 2017 International Conference on Computing, Communication and Automation (ICCCA), Greater Noida, India, 2017, pp. 1543-1548, doi: 10.1109/CCAA.2017.8230047.

[25] D. Ruhela, M. Bansal, "Vedic Multiplier with Chinese Abacus Adder Design using Reversible Logic Gates", 2nd International Conference on VLSI, Communication and Networks (VCAN-2015), pp. 9-12, 2015.

[26] M. Rawat, M. Bansal, "A Voltage mode biquad with lowpass, bandpass and notch outputs using Voltage Differencing Current Conveyor", International Journal of Advanced Research in Computer and Communication Engineering, Vol. 4, Issue 5, pp. 506-508, 2015. [27] M. Bansal, D. Ruhela, "High Speed, Energy Efficient Vedic Multiplier using Adiabatic Logic Gates", International Conference on Advances in Electrical, Power Control, Electronics \& Communication Engineering (AEPCECE-2014), 2014. 